\title{
On approximation in generalized Zygmund class
}

https://doi.org/10.1515/dema-2019-0022

Received February 24, 2019; accepted June 10, 2019

Abstract: Here, we estimate the degree of approximation of a conjugate function $\tilde{g}$ and a derived conjugate function $\tilde{g}^{\prime}$, of a $2 \pi$-periodic function $g \in Z_{r}^{\lambda}, r \geq 1$, using Hausdorff means of CFS (conjugate Fourier series) and CDFS (conjugate derived Fourier series) respectively. Our main theorems generalize four previously known results. Some important corollaries are also deduced from our main theorems. We also partially review the earlier work of the authors in respect of order of the Euler-Hausdorff product method.

Keywords: generalized Zygmund $\left(Z_{r}^{\lambda}, r \geq 1\right)$ class of function, error approximation, CFS (conjugate Fourier series), CDFS (conjugate derived Fourier series), generalized Minkowski's inequality, Hausdorff means

MSC: Primary: 42A10, 41A10; Secondary: 42B05, 42B08

\section{Introduction}

The study of error estimation of a conjugate function $\tilde{g}$ of a $2 \pi$-periodic function $g$ belonging to Lip $(\alpha)$, $\operatorname{Lip}(\alpha, r), \operatorname{Lip}(\xi(t), r)$ and $W\left(L_{r}, \xi(t)\right)$ using single summability operators such as Euler, Cesàro, Nörlund, generalized Nörlund, Hölder, Karamata, Riesz, matrix and almost matrix, has been a centre of creative study for the several researchers like Kushwaha [1], Nigam and Sharma [2], Qureshi [3-6], Lal and Nigam [7], Lal [8], Rhoades [9], Mittal et al. [10], Mishra [11] and Kranz et al. [12] in past few decades.

The study of error estimates of a conjugate function $\tilde{g}$ of a $2 \pi$-periodic function $g$ belonging to different Lipschitz classes using the products of Cesàro, Hölder and Euler has also been of great interest among the researchers like Lal and Singh [13, 14], Dhakal [15], Mishra et al. [16], Nigam and Sharma [17-20] and Padhy et al. [21] in the recent past.

It can be noted that the matrices involved in Cesàro, Hölder, Euler and their product are Hausdorff matrices ${ }^{1}$. Thus, considering this view point, Singh and Srivastava [25] studied error estimates of a conjugate function $\tilde{g}$ of a $2 \pi$-periodic function $g \in W\left(L_{r}, \xi(t)\right)$ using Hausdorff means.

Lal and Mishra [26] have obtained results on approximations of a conjugate function $\tilde{g}$ of a $2 \pi$-periodic function $g \in \operatorname{Lip} \alpha$ and $g \in \operatorname{Lip}(\xi(t), r)$ using Euler-Hausdorff means.

The review of the above research clearly suggests that the studies of error estimation of a conjugate function $\tilde{g}$ of $2 \pi$-periodic function $g \in Z_{r}^{\lambda}, r \geq 1$, using Hausdorff means of $\mathrm{CFS}^{2}$ have not been initiated so far.

Note 1: The CFS is not necessarily a FS ${ }^{3}$, for example, the series $\sum_{n=2}^{\infty} \frac{\sin (n x)}{\log n}$ conjugate to the Fourier series $\sum_{n=2}^{\infty} \frac{\cos (n x)}{\log n}$ is not a Fourier series (Zygmund [27, p. 186]).

${ }^{\star}$ Corresponding Author: Hare Krishna Nigam: Department of Mathematics., Central University of South Bihar. Gaya. Bihar. India: E-mail: hknigam@cusb.ac.in

1 The product of two Hausdorff matrices is again a Hausdorff matrix [9, 22-24].

2 CFS denotes conjugate Fourier series (Zygmund [27]) and this abbreviation will be used throughout the paper.

3 FS denotes Fourier series (Zygmund [27]) and this abbreviation will be used throughout the paper. 
In view of the above example, a separate study in case of CFS in the present direction of work is so required.

The study of $\mathrm{DFS}^{4}$ by single and product summability means has been of great interest among researchers.

A study of $\mathrm{CDFS}^{5}$ was initiated for the first time by Moursund [28] in 1935. Moursund [28] established the results on Nörlund and Cesàro means of CDFS.

Thus, in the same direction, Chandra and Dikshit [29] have studied $|B|$ and $|E, q|$ summabilities of DFS and CDFS. Lal and Nigam [30] have studied $K^{\lambda}$-summability of DFS and Lal and Yadav [31] have studied $(N, p, q)(C, 1)$ product summability of DFS.

Very recently, Mursaleen and Alotaibi [32] have studied generalized matrix summability of CDFS.

Since the studies of error estimates of a conjugate derived function $\tilde{g}^{\prime}$ of a $2 \pi$-periodic function $g$ either in the Lipschitz space or in the Zygmund space have not been initiated so far. Therefore, in this paper, we, for the very first time, also study the error estimates of a conjugate derived function $\tilde{g}^{\prime}$ of a $2 \pi$-periodic function $g \in Z_{r}^{\lambda}$, using Hausdorff means of CDFS.

A separate study in case of CDFS in the present direction is justified due to its importance in applications to science and engineering.

As the trigonometric series FS, CFS, DFS and CDFS are well known, we will not present them here. The detailed work on FS, CFS and DFS can be found in [27] and that on CDFS in [28].

We denote the $j^{\text {th }}$ partial sum of C F S as $\tilde{s}_{j}(g ; y)$, which is given by

$$
\tilde{s}_{j}(g ; y)-\tilde{g}(y)=\frac{1}{2 \pi} \int_{0}^{\pi} \gamma_{(y)}(l) \frac{\cos (j+1 / 2) l}{\sin (l / 2)} d l,
$$

where $\tilde{g}$ is the conjugate function of $2 \pi$-periodic function $g$, and is expressed as

$$
\tilde{g}(y)=-\frac{1}{2 \pi} \int_{0}^{\pi} \gamma_{(y)}(l) \cot (l / 2) d l \quad \text { (Zygmund [27, p. 50]) . }
$$

Now, we denote the $j^{\text {th }}$ partial sum of C D F S as $\tilde{s}^{\prime} j(g ; y)$, which is given by

$$
\tilde{s}_{j}^{\prime}(g ; y)-\tilde{g}^{\prime}(y)=-\frac{2}{\pi} \int_{0}^{\pi} \frac{\rho_{(y)}(l)}{4 \sin (l / 2)}\left(j+\frac{1}{2}\right) \sin \left(j+\frac{1}{2}\right) l d l-\frac{1}{\pi} \int_{0}^{\pi} \frac{\rho_{(y)}(l)}{4 \sin (l / 2)} \frac{\cos (j+1 / 2) l}{\tan (l / 2)} d l,
$$

where $\tilde{g}^{\prime}$ is the conjugate derived function of $2 \pi$-periodic function $g$, which is expressed as

$$
\tilde{g}^{\prime}(y)=\frac{1}{4 \pi} \int_{0}^{\pi} \operatorname{cosec}^{2} \frac{l}{2} \rho_{(y)}(l) d l \quad \text { (Moursund [28]) . }
$$

Note 2: For better understanding, formulas (3) and (4) are derived in the proof of Theorem 2. Here,

$$
\gamma_{(y)}(l)=g(y+l)-g(y-l)
$$

and

$$
\rho_{(y)}(l)=g(y+l)+g(y-l) \text { (Zygmund [27]). }
$$

We mention here some standard inequalities which are used in the paper:

$$
\frac{1}{\sin (l / 2)} \leq \frac{\pi}{l}, \quad 0<l \leq \pi
$$

\footnotetext{
4 DFS denotes derived Fourier series (Zygmund [27]) and this abbreviation will be used throughout the paper.

5 CDFC denotes conjugate derived Fourier series (Moursund [28]) and this abbreviation will be used throughout the paper.
} 


$$
\begin{gathered}
\sin l \leq l, \quad l \geq 0 ; \\
|\sin l| \leq 1, \quad|\cos l| \leq 1, \quad \text { for all } l .
\end{gathered}
$$

In 1921, Hausdorff [33] gave the following definition:

A Hausdorff matrix $H \equiv\left(\Theta_{j, a}\right)$ is an infinite lower triangular matrix with nonzero entries

$$
\Theta_{j, a}=\left\{\begin{array}{ll}
\left(\begin{array}{c}
j \\
a
\end{array}\right) \Delta^{j-a} \mu_{a} & 0 \leq a \leq j \\
0 & a>j
\end{array} ;\right.
$$

where $\Delta$ is a forward difference operator defined by $\Delta \mu_{j}=\mu_{j}-\mu_{j+1}$ and $\Delta^{a+1} \mu_{j}=\Delta^{a}\left(\Delta \mu_{j}\right)$. If $H$ is regular, then $\mu_{j}$ is known as a moment sequence. $\mu_{j}$ has the representation $\mu_{j}=\int_{0}^{1} v^{j} d \zeta(v)$, where $\zeta(v)$ is known as the mass function. $\zeta(v)$ is continuous at $v=0$ and it belongs to $B V[0,1]$ such that $\zeta(0)=0, \zeta(1)=1$; and for $0<v<1, \zeta(v)=[\zeta(v+0)+\zeta(v-0)] / 2$.

The Hausdorff means $H$ of CFS is given by

$$
\tilde{M}_{j}^{H}(g ; y):=\sum_{a=0}^{j} \Theta_{j, a} \tilde{s}_{a}(g ; y), \quad j=0,1,2, \ldots
$$

If $\tilde{M}_{j}^{H}(g ; y) \rightarrow s$ as $j \rightarrow \infty$, then the CFS is said to be summable to $s$ by the $H$ method [34].

Note 3: A detailed study of Hausdorff matrices can be found in [23, 35].

\section{Particular cases of Hausdorff means:}

Hausdorff means have following particular cases:

(i) Cesàro means $(C, m)$ is a Hausdorff method with the mass function $\zeta(v)=m \int_{0}^{v}(1-l)^{m-1} d l$.

(ii) Hölder means $(H, m)$ is a Hausdorff method with the mass function $\zeta(v)=\int_{0}^{v} \frac{1}{(m-1) !}\left(\log \left(\frac{1}{l}\right)\right)^{m-1} d l[24$, pp. 39-40].

(iii) For the mass function

$$
\zeta(v)= \begin{cases}0, & \text { if } v \in[0, b] \\ 1, & \text { if } v \in[b, 1],\end{cases}
$$

where $b=1 /(1+q), q>0$, the Hausdorff method $H \equiv\left(\Theta_{j, a}\right)$ is the Euler means $(E, q), q>0$.

Remark 1. As per the above discussion of particular cases of Hausdorff means, our main results also hold for Cesàro, Euler and Hölder means, as well as their product methods.

In view of Remark 1, the products of Euler and Cesàro are again Hausdorff matrices [9, 22-24]. Note that we need to check the order of the product of Euler and Cesàro means. Now a question arises: Is the $(E, q)$ method generated from the Hausdorff moment sequence $\zeta_{j}=\frac{1}{(q+1)^{j}}$ a right or left shifting sequence of moments. Similarly, is the $(C, 1)$ method generated from the Hausdorff moment sequence $\zeta_{j}=\frac{1}{j+1}$ a right or left shifting sequence of moments.

Lal and Mishra [26] have considered the $(E, q)$-Hausdorff product transform and have reduced the Hausdorff matrix to a $(C, 1)$ matrix as a right shifting sequence (see corollary 8.3 of [26, p. 12]), which appears false because a $(C, 1)$ matrix is a left shifting sequence as defined in Keska [36, p. 68]. Infact, Keska [36] gave the following:

Definition 1. [36, p. 67] A moment sequence $\zeta_{j}$ is known as a right-shifting sequence of moments if there exists $\zeta_{0}^{\star} \in \mathbb{R}$ such that the sequence

$$
\zeta_{0}^{\star}, \zeta_{1}^{\star}=\zeta_{0}, \ldots, \zeta_{k+1}^{*}=\zeta_{k}^{\star}, \ldots
$$


is a moment sequence

$$
\zeta_{j}=\int_{0}^{1} x^{j} d \chi(x)
$$

where $\chi$ is a real, bounded variation function defined on the interval $[0,1]$ satisfying the conditions

$$
\chi(0+)=\chi(0)=0 \text { and } \chi(1)=1 .
$$

Example 1. [36, p. 68] The Hausdorff moment sequence $\zeta_{j}=\frac{1}{(q+1)^{j}}$, which generates the Euler $\operatorname{method}(E, q)$, is a right-shifting sequence of moments because $\Delta^{j} \zeta_{0}^{\star}=(1+q) \Delta^{j} \zeta_{0} \geq 0$ for $\zeta_{0}^{\star}=(1+q)$.

Example 2. [36, p. 68] The Haudsorff moment sequence $\zeta_{j}=\frac{1}{j+1}$, which generates the Cesàro method $C^{1}$, is not a right-shifting sequence of moments.

Remark 2. In view of above facts, Corollary 8.3 of [26, p. 12] cannot be reduced to the method $(E, 1)(C, 1)$ unless $(C, 1)$ is considered as a left shifting sequence. Also, Corollary 8.4-8.6 of [26, p. 13] cannot be reduced to the methods $(E, q)(C, m),(E, q)(C, 1),(E, 1)(C, m)$ or $(E, 1)(C, 1)$ unless $(C, m)$ or $(C, 1)$ is considered as a left shifting sequence.

Let

$$
L^{r}[0,2 \pi]:=\left\{g:[0,2 \pi] \rightarrow \mathbb{R}: \int_{0}^{2 \pi}|g(y)|^{r} d y<\infty\right\}, \quad r \geq 1,
$$

be the space of $2 \pi$-periodic and integrable functions.

We define $\|\cdot\|_{r}$ by

$$
\|g\|_{r}:= \begin{cases}\left\{\frac{1}{2 \pi} \int_{0}^{2 \pi}|g(y)|^{r} d y\right\}^{1 / r} & \text { for } 1 \leq r<\infty ; \\ e s s \sup _{y \in(0,2 \pi)}|g(y)| & \text { for } r=\infty .\end{cases}
$$

Now, we define

$$
Z_{r}^{(\lambda)}:=\left\{g \in L^{r}[0,2 \pi]: r \geq 1, \sup _{l \neq 0} \frac{\|g(\cdot+l)-g(\cdot-l)\|_{r}}{\lambda(l)}<\infty\right\}
$$

and a norm $\|g\|_{r}^{(\lambda)}$ on $Z_{r}^{(\lambda)}$ is defined by

$$
\|g\|_{r}^{(\lambda)}:=\|g\|_{r}+\sup _{l \neq 0} \frac{\|g(\cdot+l)-g(\cdot-l)\|_{r}}{\lambda(l)}, \quad r \geq 1,
$$

where $\lambda:[0,2 \pi] \rightarrow \mathbb{R}$ is an arbitrary function with $\lambda(l)>0$ for $0<l \leq 2 \pi$ and $\lim _{l \rightarrow 0+} \lambda(l)=\lambda(0)=0$. (Zygmund [27])

The completeness of the space $Z_{r}^{(\lambda)}$ can be discussed by considering the completeness of $L^{r}, r \geq 1$. Hence the space $Z_{r}^{\lambda}$ is a Banach space under the norm $\|\cdot\|_{r}^{(\lambda)}$.

Remark 3. Throughout the paper, $\lambda$ and $v$ denote moduli of continuity of order two such that $\frac{\lambda(l)}{v(l)}$ is positive and non-decreasing in $l$, then

$$
\|g\|_{r}^{(v)} \leq \max \left(1, \frac{\lambda(2 \pi)}{v(2 \pi)}\right)\|g\|_{r}^{(\lambda)}<\infty .
$$

Thus,

$$
Z_{r}^{(\lambda)} \subset Z_{r}^{(v)} \subset L^{r}, r \geq 1 .
$$


Remark 4. Any modulus of continuity of first order $\lambda=\lambda(g, \cdot)$ satisfies the following conditions:

(i) $\lambda(0)=0$;

(ii) the function $\lambda$ is continuous on $[0,+\infty)$;

(iii) the function $\lambda$ is non-decreasing on $[0,+\infty)$;

(iv) the function $\lambda$ is semiadditive; i. e. the inequality $\lambda\left(l_{1}+l_{2}\right) \leq \lambda\left(l_{1}\right)+\lambda\left(l_{2}\right)$ holds for any $l_{1} \geq 0$ and $l_{2} \geq 0$.

Conversely, if a function $\lambda$ satisfies conditions (i)-(iv), then it is the first order modulus of continuity of the function $g(y)=\lambda(|y|)$. Moreover, it can be easily seen that $\lambda$ is the second order modulus of continuity of the function $g(y)=\frac{\lambda(|y|)}{2}$. If a function $\lambda$ satisfies conditions (i)-(iii) and the function $\frac{\lambda(l)}{l}$ is non-increasing on $(0,+\infty)$, then the semiadditivity condition (iv) also holds, and so $\lambda$ is the modulus of continuity of the first and second order for some continuous functions.

The modulus of continuity of second order satisfies conditions (i)-(iii) and a further condition, given as follows:

(v) the inequality $\lambda(n l) \leq n^{2} \lambda(l)$ holds for any $l \geq 0$ and $n \in N$.

Geit [37] constructed a wide class of the functions that are second-order moduli of continuity of $2 \pi$ periodic functions. It can be easily shown that condition (v) for non-negative functions follows from the following condition:

(vi) the function $\frac{\lambda(l)}{l^{2}}$ is non-increasing on $(0,+\infty)$.

Note 4: Readers may refer to the paper of Konyagin [38] in support of Remark 4. Readers may also refer to the paper of Weiss and Zygmund [39] which dealt with conditions on the second order modulus of smoothness sufficient to force absolute continuity of a function. The technique employed in [39] is nearly identical to that of [40].

\section{Remark 5.}

(i) If we take $r \rightarrow \infty$ in $Z_{r}^{(\lambda)}$, then $Z_{r}^{(\lambda)}$ reduces to $Z^{(\lambda)}$.

(ii) If we take $\lambda(l)=l^{\alpha}$ in $Z^{(\lambda)}$, then $Z^{(\lambda)}$ reduces to $Z_{\alpha}$.

(iii) If we take $\lambda(l)=l^{\alpha}$ in $Z_{r}^{(\lambda)}$, then $Z_{r}^{(\lambda)}$ reduces to $Z_{\alpha, r}$.

(iv) If we take $r \rightarrow \infty$ in $Z_{\alpha, r}$, then $Z_{\alpha, r}$ reduces to $Z_{\alpha}$.

(v) Let $0 \leq \alpha_{1}<\alpha_{2}<1$. If $\lambda(l)=l^{\alpha_{2}}$ and $\quad v(l)=l^{\alpha_{1}}$, then $\lambda(l) / v(l)$ is increasing, but $\lambda(l) / l v(l)$ is a decreasing function of $l$.

We write

$$
\begin{aligned}
& f(v, l)=\operatorname{Re}\left[\sum_{a=0}^{j}\left(\begin{array}{c}
j \\
a
\end{array}\right) v^{a}(1-v)^{j-a} e^{i(a+1 / 2) l}\right] ; \\
& f_{1}(v, l)=\operatorname{Im}\left[\sum_{a=0}^{j}\left(\begin{array}{c}
j \\
a
\end{array}\right) v^{a}(1-v)^{j-a} e^{i(a+1 / 2) l}\right] ; \\
& f_{2}(v, l)=\operatorname{Re}\left[\sum_{a=0}^{j}\left(\begin{array}{c}
j \\
a
\end{array}\right) v^{a}(1-v)^{j-a} e^{i a l}\right] ; \\
& \tilde{Y}_{j}^{H}(l)=\frac{1}{2 \pi \sin (l / 2)} \int_{0}^{1} f(v, l) d \zeta(v) ; \\
& \tilde{Y}_{1 j}^{\prime H}(l)=-\frac{a}{2 \pi \sin ^{H}(l / 2)} \int_{0}^{1} f_{1}(v, l) d \zeta(v) ; \\
& \tilde{Y}_{2 j}^{\prime H}(l)=-\frac{1}{4 \pi \sin ^{2}(l / 2)} \int_{0}^{1} f_{2}(v, l) d \zeta(v) .
\end{aligned}
$$




\section{The Main Theorems}

Theorem 1. Error approximation of a conjugate function $\tilde{g}$ of $2 \pi$-periodic function $\quad g \in Z_{r}^{(\lambda)}, r \geq 1$, using $H \equiv\left(\Theta_{j, a}\right)$ of CFS is given by

$$
\left\|\tilde{M}_{j}^{H}(g, \cdot)-\tilde{g}(\cdot)\right\|_{r}^{(v)}=O\left(\frac{1}{j+1} \int_{\frac{1}{j+1}}^{\pi} \frac{\lambda(l)}{l^{2} v(l)} d l\right),
$$

where $\lambda(l)$ and $v(l)$ are as defined in Remark 3, provided

$$
\int_{0}^{\eta} \frac{\lambda(l)}{l v(l)} d l=O\left(\frac{\lambda(\eta)}{v(\eta)}\right), \quad 0<\eta<\pi .
$$

Theorem 2. Error approximation of a conjugate derived conjugate function $\tilde{g^{\prime}}$ of a $2 \pi$-periodic function $g \in$ $Z_{r}^{(\lambda)}, r \geq 1$, using $H \equiv\left(\Theta_{j, a}\right)$ of CDFS is given by

$$
\left\|\tilde{M}_{j}^{\prime}{ }_{j}(g, \cdot)-\tilde{g}^{\prime}(\cdot)\right\|_{r}^{v}=O\left(\frac{1}{j+1} \int_{\frac{1}{j+1}}^{\pi} \frac{(l+1) \lambda(l)}{l^{3} v(l)}\right),
$$

where $\lambda(l)$ and $v(l)$ are as defined in Remark 3, provided

$$
\int_{0}^{\eta} \frac{\lambda(l)}{l^{2} v(l)} d l=O\left(\frac{1}{\eta} \frac{\lambda(\eta)}{v(\eta)}\right), \quad 0<\eta<\pi .
$$

\section{Lemmas}

We use the following lemmas:

Lemma 1. For $l \in\left(0, \frac{1}{j+1}\right], v \in[0,1]$,

$$
\left|\int_{0}^{1} f(v, l) d \zeta(v)\right|=O(1)
$$

Lemma 2. For $l \in\left[\frac{1}{j+1}, \pi\right], v \in[0,1]$,

$$
\left|\int_{0}^{1} f(v, l) d \zeta(v)\right|=O\left(\frac{1}{l(j+1)}\right) .
$$

Lemmas 1 and 2 are proved using similar arguments to those used in the proofs of Singh and Srivastava ([25], Lemma 1).

Lemma 3.

$$
\left|\tilde{Y}_{j}^{H}(l)\right|= \begin{cases}O\left(\frac{1}{l}\right), & l \in(0,1 /(j+1)] ; \\ O\left(1 / l^{2}(j+1)\right), & l \in[1 /(j+1), \pi] .\end{cases}
$$

Proof. Using (5) and above Lemmas 1 and 2, Lemma 3 can be obtained directly. 
Lemma 4. [41, p. 3]: For $0 \leq v \leq 1$ and $0 \leq l \leq \pi$,

$$
\left|\int_{0}^{1} f(v, l) d \zeta(v)\right|= \begin{cases}O((j+1) l), & l \in\left[0, \frac{1}{j+1}\right] \\ O\left((j+1)^{-1} l^{-1}\right), & l \in\left[\frac{1}{j+1}, \pi\right] .\end{cases}
$$

Lemma 5.

$$
\left|\tilde{Y}_{1 j}^{\prime}(l)\right|= \begin{cases}O(j+1), & l \in\left(0, \frac{1}{j+1}\right] ; \\ O\left((j+1)^{-1} l^{-2}\right), & l \in\left[\frac{1}{j+1}, \pi\right] .\end{cases}
$$

Proof. Using (5) and above Lemma 4, Lemma 5 can be obtained directly.

Lemma 6. For $l \in\left(0, \frac{1}{j+1}\right], v \in[0,1]$,

$$
\left|\int_{0}^{1} f_{2}(v, l) d \zeta(v)\right|=O(1)
$$

Proof. We can write

$$
f_{2}(v, l)=(1-v)^{j} \operatorname{Re}\left[\sum_{a=0}^{j} j C_{a}\left(\frac{v e^{i l}}{1-v}\right)^{a}\right]=\operatorname{Re}\left[\left(1-v+v e^{i l}\right)^{j}\right], \quad \text { continuous for } v \in[0,1] .
$$

If $K=\sup _{v \in[0,1]} \frac{d \zeta(v)}{d v}$, then

$$
\begin{aligned}
\int_{0}^{1} f_{2}(v, l) d \zeta(v) \leq & K \int_{0}^{1} f_{2}(v, l) d v \\
& =O(1) \int_{0}^{1} \operatorname{Re}\left[\left(1-v+v e^{i l}\right)^{j}\right] d v \\
& =O(1) \operatorname{Re}\left[\frac{\left(1-v+v e^{i l}\right)^{j+1}}{\left(e^{i l}-1\right)(j+1)}\right]_{v=0}^{1} \\
& =O(1) \operatorname{Re}\left[\frac{e^{i l(j+1)}-1}{\left(e^{i l}-1\right)(j+1)}\right] \\
& =O(1) \operatorname{Re}\left[\frac{\cos (j+1) l+i \sin (j+1) l-1}{(\cos l+i \sin l-1)(j+1)}\right] \\
& =O\left(\frac{1}{j+1}\right) \operatorname{Re}\left[\frac{\cos (j+1) l-1}{\cos l-1-i \sin l\left\{(\cos l-1)^{2}+\sin ^{2} l\right\}}+\frac{i \sin (j+1) l\{(\cos l-1)-i \sin l\}}{\left\{(\cos l-1)^{2}+\sin ^{2} l\right\}}\right] \\
& =O\left(\frac{1}{j+1}\right)\left[\frac{\cos (j+1) l \cos l-\cos (j+1) l-\cos l+1}{2(1-\cos l)}+\frac{\sin (j+1) l \sin l}{2(1-\cos l)}\right] .
\end{aligned}
$$

Using trigonometric properties, we get

$$
\begin{aligned}
\int_{0}^{1} f_{2}(v, l) d \zeta(v) & =O\left(\frac{1}{j+1}\right)\left[\frac{\cos j l-\cos (j+1) l-\cos l+1}{2(1-\cos l)}\right] \\
& =O\left(\frac{1}{j+1}\right)\left[\frac{2 \sin (j+1 / 2) l \sin (l / 2)+2 \sin ^{2}(l / 2)}{4 \sin ^{2}(l / 2)}\right] \\
& =O\left[\frac{\sin (j+1 / 2) l+\sin (l / 2)}{2(j+1) \sin (l / 2)}\right]
\end{aligned}
$$




$$
=O\left[\frac{\sin ((j+1) l / 2) \cos (j l / 2)}{(j+1) \sin (l / 2)}\right] .
$$

Using (5), (6) and (7), we get Lemma 6.

Lemma 7. For $l \in\left[\frac{1}{j+1}, \pi\right], v \in[0,1]$,

$$
\left|\int_{0}^{1} f_{2}(v, l) d \zeta(v)\right|=O\left(\frac{1}{l(j+1)}\right) .
$$

Proof. Using (5) and (7), we get Lemma 7.

Lemma 8.

$$
\left|\tilde{Y}_{2 j}^{\prime H}(l)\right|= \begin{cases}O\left(l^{-2}\right), & l \in\left(0, \frac{1}{j+1}\right] ; \\ O\left((j+1)^{-1} l^{-3}\right), & l \in\left[\frac{1}{j+1}, \pi\right] .\end{cases}
$$

Proof. Using (5), Lemmas 6 and 7, we get Lemma 8.

\section{Proof of the Main Theorems}

\subsection{Proof of Theorem 1}

Using (1),

$$
\begin{aligned}
\tilde{M}_{j}^{H}(g, y)-\tilde{g}(y) & =\sum_{a=0}^{j} \Theta_{j, a}\left\{\tilde{s}_{a}(g ; y)-\tilde{g}(y)\right\}=\frac{1}{2 \pi} \int_{0}^{\pi}\left(\frac{\gamma_{(y)}(l)}{\sin (l / 2)} \sum_{a=0}^{j}\left(\begin{array}{c}
j \\
a
\end{array}\right) \Delta^{j-a} \mu_{a} \cos (a+1 / 2) l\right) d l \\
& =\frac{1}{2 \pi} \int_{0}^{\pi}\left[\left(\frac{\gamma_{(y)}(l)}{\sin (l / 2)} \sum_{a=0}^{j}\left(\begin{array}{c}
j \\
a
\end{array}\right)\right)\left(\int_{0}^{1} v^{a}(1-v)^{j-a} d \zeta(v) \operatorname{Re}\left\{e^{i(a+1 / 2) l}\right\}\right)\right] d l \\
& =\frac{1}{2 \pi} \int_{0}^{\pi}\left[\left(\frac{\gamma_{(y)}(l)}{\sin (l / 2)}\right)\left(\int_{0}^{1} \operatorname{Re}\left[\sum_{a=0}^{j}\left(\begin{array}{c}
j \\
a
\end{array}\right) v^{a}(1-v)^{j-a} e^{i(a+1 / 2) l}\right] d \zeta(v)\right)\right] d l \\
& =\frac{1}{2 \pi} \int_{0}^{\pi}\left(\frac{\gamma_{(y)}(l)}{\sin (l / 2)} \int_{0}^{1} f(v, l) d \zeta(v)\right) d l \\
& =\int_{0}^{\pi} \gamma_{(y)}(l) \tilde{Y}_{j}^{H}(l) d l .
\end{aligned}
$$

Let

$$
k_{j}(y):=\tilde{M}_{j}^{H}(g, y)-\tilde{g}(y)=\int_{0}^{\pi} \gamma_{(y)}(l) \tilde{Y}_{j}^{H}(l) d l
$$

Then

$$
k_{j}(y+z)-k_{j}(y-z)=\int_{0}^{\pi}\left[\gamma_{(y+z)}(l)-\gamma_{(y-z)}(l)\right] \tilde{Y}_{j}^{H}(l) d l .
$$

Using the generalized Minkowski inequality (Chui [42]),

$$
\left\|k_{j}(\cdot+z)-k_{j}(\cdot-z)\right\|_{r}=\left\{\frac{1}{2 \pi} \int_{0}^{2 \pi}\left|k_{j}(y+z)-k_{j}(y-z)\right|^{r} d y\right\}^{1 / r}
$$




$$
\begin{aligned}
& \leq \int_{0}^{\pi}\left\{\frac{1}{2 \pi} \int_{0}^{2 \pi}\left|\left\{\gamma_{(y+z)}(l)-\gamma_{(y-z)}(l)\right\}\right|^{r} d y\right\}^{\frac{1}{r}}\left|\tilde{Y}_{j}^{H}(l)\right| d l \\
& =\int_{0}^{1 /(j+1)}\left\|\gamma_{(\cdot+z)}(l)-\gamma_{(\cdot-z)}(l)\right\|_{r}\left|\tilde{Y}_{j}^{H}(l)\right| d l+\int_{1 /(j+1)}^{\pi}\left\|\gamma_{(\cdot+z)}(l)-\gamma_{(\cdot-z)}(l)\right\|_{r}\left|\tilde{Y}_{j}^{H}(l)\right| d l \\
& :=I_{1}+I_{2} \quad \text { (say). }
\end{aligned}
$$

Further,

$$
\left|\gamma_{(y+z)}(l)-\gamma_{(y-z)}(l)\right| \leq|g(y+z+l)-g(y+z-l)|+|g(y-z+l)-g(y-z-l)| .
$$

Applying Minkowski's inequality (Zygmund [27]),

$$
\left\|\gamma_{(\cdot+z)}(l)-\gamma_{(\cdot-z)}(l)\right\|_{r} \leq\|g(\cdot+z+l)-g(\cdot+z-l)\|_{r}+\|g(\cdot-z+l)-g(\cdot-z-l)\|_{r}=O(\lambda(l)) .
$$

Also

$$
\left\|\gamma_{(\cdot+z)}(l)-\gamma_{(\cdot-z)}(l)\right\|_{r} \leq\|g(\cdot+l+z)-g(\cdot+l-z)\|_{r}+\|g(\cdot-l+z)-g(\cdot-l-z)\|_{r}=O(\lambda(z)) .
$$

Since $v(l)$ is positive and non-decreasing and for $l \leq z$, we have

$$
\left\|\gamma_{(\cdot+z)}(l)-\gamma_{(\cdot-z)}(l)\right\|_{r}=O(\lambda(l))=O\left(v(l)\left(\frac{\lambda(l)}{v(l)}\right)\right)=O\left(v(z)\left(\frac{\lambda(l)}{v(l)}\right)\right) .
$$

Since $\lambda(l) / v(l)$ is positive and increasing, and if $l \geq z$, then $\lambda(l) / v(l) \geq \lambda(z) / v(z)$ and we have

$$
\left\|\gamma_{(\cdot+z)}(l)-\gamma_{(\cdot-z)}(l)\right\|_{r}=O(\lambda(z))=O\left(v(z)\left(\frac{\lambda(l)}{v(l)}\right)\right) .
$$

Using (9), (16), Lemma 3 and $\lambda(l) / v(l)$ being monotonic with $l$, we have

$$
I_{1}=\int_{0}^{1 /(j+1)}\left\|\gamma_{(\cdot+z)}(l)-\gamma_{(\cdot-z)}(l)\right\|_{r}\left|\tilde{Y}_{j}^{H}(l)\right| d l=O\left(\int_{0}^{1 /(j+1)} v(z) \frac{\lambda(l)}{v(l)} \frac{d l}{l}\right)=O\left(v(z) \frac{\lambda(1 /(j+1))}{v(1 /(j+1))}\right) .
$$

Also, using Lemma 3 and (16),

$$
I_{2}=\int_{1 /(j+1)}^{\pi}\left\|\gamma_{(\cdot+z)}(l)-\gamma_{(\cdot-z)}(l)\right\|_{r}\left|\tilde{Y}_{j}^{H}(l)\right| d l=O\left(\int_{1 /(j+1)}^{\pi} v(z) \frac{\lambda(l)}{v(l)} \frac{d l}{l^{2}(j+1)}\right)=O\left(\frac{v(z)}{j+1} \int_{1 /(j+1)}^{\pi} \frac{\lambda(l)}{l^{2} v(l)} d l\right) .
$$

By (12), (17) and (18),

$$
\left\|k_{j}(\cdot+z)-k_{j}(\cdot-z)\right\|_{r}=O\left(v(z) \frac{\lambda\left((j+1)^{-1}\right)}{v\left((j+1)^{-1}\right)}\right)+O\left(\frac{v(z)}{j+1} \int_{1 /(j+1)}^{\pi} \frac{\lambda(l)}{l^{2} v(l)} d l\right) .
$$

Thus,

$$
\sup _{z \neq 0} \frac{\left\|k_{j}(\cdot+z)-k_{j}(\cdot-z)\right\|_{r}}{v(z)}=O\left(\frac{\lambda\left((j+1)^{-1}\right)}{v\left((j+1)^{-1}\right)}\right)+O\left(\frac{1}{j+1} \int_{1 /(j+1)}^{\pi} \frac{\lambda(l)}{l^{2} v(l)} d l\right) .
$$

Since $g \in Z_{r}^{(\lambda)}$, then

$$
\left\|\gamma_{(\cdot)}(l)\right\|_{r}=\|g(\cdot+l)-g(\cdot-l)\|_{r}=O(\lambda(l)) .
$$

From (9), when $v(l)=1$, we get

$$
\int_{0}^{\eta} \frac{\lambda(l)}{l} d l=O(\lambda(\eta)) \quad 0<\eta<\pi
$$


Using (20), (21) and Lemma 3,

$$
\begin{aligned}
\left\|k_{j}(\cdot)\right\|_{r} & =\left(\int_{0}^{1 /(j+1)}+\int_{1 /(j+1)}^{\pi}\right)\left\|\gamma_{(\cdot)}(l)\right\|_{r}\left|\tilde{Y}_{j}^{H}(l)\right| d l \\
& =\int_{0}^{1 /(j+1)}\left\|\gamma_{(\cdot)}(l)\right\|_{r}\left|\tilde{Y}_{j}^{H}(l)\right| d l+\int_{1 /(j+1)}^{\pi}\left\|\gamma_{(\cdot)}(l)\right\|_{r}\left|\tilde{Y}_{j}^{H}(l)\right| d l \\
& =O\left(\int_{0}^{1 /(j+1)} \frac{\lambda(l)}{l} d l\right)+O\left(\int_{1 /(j+1)}^{\pi} \frac{\lambda(l)}{l^{2}(j+1)} d l\right) \\
& =O(\lambda(1 /(j+1)))+O\left(\int_{1 /(j+1)}^{\pi} \frac{\lambda(l)}{l^{2}(j+1)} d l\right) \\
& =O(\lambda(1 /(j+1)))+O\left(\frac{1}{j+1} \int_{1 /(j+1)}^{\pi} \frac{\lambda(l)}{l^{2}} d l\right) .
\end{aligned}
$$

Now, by (19) and (22), we have

$$
\begin{aligned}
\left\|k_{j}(\cdot)\right\|_{r}^{(v)} & =\left\|k_{j}(\cdot)\right\|_{r}+\sup _{z \neq 0} \frac{\left\|k_{j}(\cdot+z)-k_{j}(\cdot-z)\right\|_{r}}{v(z)} \\
& =O\left(\lambda\left((j+1)^{-1}\right)\right)+O\left(\frac{1}{j+1} \int_{1 /(j+1)}^{\pi} \frac{\lambda(l)}{l^{2}} d l\right)+O\left(\frac{\lambda\left((j+1)^{-1}\right)}{v\left((j+1)^{-1}\right)}\right)+O\left(\frac{1}{j+1} \int_{1 /(j+1)}^{\pi} \frac{\lambda(l)}{l^{2} v(l)} d l\right) \\
& =\sum_{i=1}^{4} O\left(S_{i}\right) \text { (say). }
\end{aligned}
$$

Now, we write $S_{1}, S_{2}$ and $S_{3}$ in terms of $S_{4}$. Since, we have $\lambda(l)=\frac{\lambda(l)}{v(l)} \cdot v(l) \leq \frac{\lambda(l)}{v(l)} v(\pi), 0<l \leq \pi$, then

$$
S_{1}=O\left(S_{3}\right)
$$

and

$$
S_{2}=\left(\frac{1}{(j+1)} \int_{1 /(j+1)}^{\pi} \frac{\lambda(l)}{l^{2} v(l)} v(l) d l\right) \leq\left(\frac{v(\pi)}{(j+1)} \int_{1 /(j+1)}^{\pi} \frac{\lambda(l)}{l^{2} v(l)} d l\right)=O\left(S_{4}\right) .
$$

Since $\lambda(l) / v(l)$ is positive and increasing where $\lambda(l)$ and $v(l)$ are as in Remark 3, then

$$
\begin{aligned}
S_{4} & =\frac{1}{(j+1)} \int_{1 /(j+1)}^{\pi} \frac{\lambda(l)}{l^{2} v(l)} d l \geq \frac{\lambda(1 /(j+1))}{v(1 /(j+1))} \frac{1}{(j+1)} \int_{1 /(j+1)}^{\pi} \frac{d l}{l^{2}} \\
& =\frac{\lambda(1 /(j+1))}{v(1 /(j+1))} \frac{1}{(j+1)}\left[-\frac{1}{l}\right]_{1 /(j+1)}^{\pi}=\frac{\lambda(1 /(j+1))}{v(1 /(j+1))} \frac{1}{(j+1)}\left[j+1-\frac{1}{\pi}\right] \geq \frac{\lambda(1 /(j+1))}{v(1 /(j+1))} \frac{j}{(j+1)} \geq \frac{\lambda(1 /(j+1))}{2 v(1 /(j+1))} .
\end{aligned}
$$

Then,

$$
S_{3}=O\left(S_{4}\right) .
$$

From (23), (24) and (25),

$$
\left\|k_{j}(\cdot)\right\|_{r}^{(v)}=\sum_{i=1}^{4} O\left(S_{i}\right)=O\left(S_{4}\right)=O\left(\frac{1}{(j+1)} \int_{1 /(j+1)}^{\pi} \frac{\lambda(l)}{l^{2} v(l)} d l\right) .
$$

Hence

$$
\left\|\tilde{M}_{j}^{H}(g, \cdot)-\tilde{g}(\cdot)\right\|_{r}=O\left(\frac{\int_{1 /(j+1) \frac{\lambda(l)}{l} l^{2} v(l)}^{\pi} d l}{j+1}\right) .
$$




\subsection{Proof of Theorem 2}

The $j^{\text {th }}$ partial sum of CDFS is given by

$$
\begin{aligned}
\tilde{s}_{j}^{\prime}(g ; y)= & \frac{1}{\pi} \int_{-\pi}^{\pi} g(u) \frac{\partial}{\partial y}\left(\sum_{m=1}^{a} \sin m(u-y)\right) d u \\
= & -\frac{1}{\pi} \int_{0}^{\pi} \frac{d}{d l}\left(\frac{\cos (l / 2)-\cos (a+1 / 2) l}{2 \sin (l / 2)}\right)\{g(y+l)+g(y-l)\} d l \\
= & -\frac{1}{\pi} \int_{0}^{\pi} \frac{d}{d l}\left(\frac{1}{2} \cos \frac{l}{2}\right) \rho_{(y)}(l) d l+\frac{1}{\pi} \int_{0}^{\pi} \frac{d}{d l}\left(\frac{\cos (a+1 / 2) l}{2 \sin (l / 2)}\right) \rho_{(y)}(l) d l \\
= & \frac{1}{4 \pi} \int_{0}^{\pi} \operatorname{cosec}^{2} \frac{l}{2} \rho_{(y)}(l) d l \\
& -\frac{2}{\pi} \int_{0}^{\pi} \frac{\rho_{(y)}(l)}{4 \sin (l / 2)}\left(a+\frac{1}{2}\right) \sin \left(a+\frac{1}{2}\right) l d l-\frac{1}{\pi} \int_{0}^{\pi} \frac{\rho_{(y)}(l)}{4 \sin (l / 2)} \frac{\cos (a+1 / 2) l}{\tan (l / 2)} d l .
\end{aligned}
$$

Writing $\varphi_{(y)}(l)=\frac{\rho_{(y)}(l)}{4 \sin (l / 2)}$, we have

$$
\begin{aligned}
\tilde{s}_{j}^{\prime}(g ; y)-\frac{1}{4 \pi} \int_{0}^{\pi} \operatorname{cosec}^{2} \frac{l}{2} \rho_{(y)}(l) d l \tilde{g}^{\prime}(y)= & -\frac{2}{\pi}\left(a+\frac{1}{2}\right) \int_{0}^{\pi} \varphi_{(y)}(l) \sin \left(a+\frac{1}{2}\right) l d l \\
& -\frac{1}{\pi} \int_{0}^{\pi} \varphi_{(y)}(l) \frac{\cos (a+1 / 2) l \cos (l / 2)}{\sin (l / 2)} d l \\
\tilde{s}_{j}{ }_{j}(g ; y)-\tilde{g}^{\prime}(y)= & {\left[-\frac{2 a}{\pi} \int_{0}^{\pi} \varphi_{(y)}(l) \sin \left(a+\frac{1}{2}\right) l d l-\frac{1}{\pi} \int_{0}^{\pi}\left(\varphi_{(y)}(l) \frac{\sin (a+1 / 2) l \sin (l / 2)+\cos (a+1 / 2) l \cos (l / 2)}{\sin (l / 2)}\right) d l\right] } \\
= & -\frac{2 a}{\pi} \int_{0}^{\pi} \varphi_{(y)}(l) \sin \left(a+\frac{1}{2}\right) l d l-\frac{1}{\pi} \int_{0}^{\pi} \varphi_{(y)}(l) \frac{\cos a l}{\sin (l / 2)} d l .
\end{aligned}
$$

Further,

$$
\begin{aligned}
\tilde{M}_{j}^{\prime H}(y)-\tilde{g}^{\prime}(y)= & \sum_{a=0}^{j} \Theta_{j, a}\left\{\tilde{s}^{\prime} a(g ; y)-\tilde{g}^{\prime}(y)\right\} \\
= & -\frac{2 a}{\pi} \int_{0}^{\pi} \varphi_{(y)}(l) \sum_{a=0}^{j} \Theta_{j, a} \sin \left(a+\frac{1}{2}\right) l d l-\frac{1}{\pi} \int_{0}^{\pi} \varphi_{(y)}(l) \sum_{a=0}^{j} \Theta_{j, a} \frac{\cos a l}{\sin (l / 2)} d l \\
= & -\frac{2 a}{\pi} \int_{0}^{\pi} \varphi_{(y)}(l) \sum_{a=0}^{j}\left(\begin{array}{c}
j \\
a
\end{array}\right) \Delta^{j-a} \mu_{a} \sin \left(a+\frac{1}{2}\right) l d l-\frac{1}{\pi} \int_{0}^{\pi} \varphi_{(y)}(l) \sum_{a=0}^{j}\left(\begin{array}{c}
j \\
a
\end{array}\right) \Delta^{j-a} \mu_{a} \frac{\cos a l}{\sin (l / 2)} d l \\
= & -\frac{2 a}{\pi} \int_{0}^{\pi}\left(\varphi_{(y)}(l) \sum_{a=0}^{j}\left(\begin{array}{c}
j \\
a
\end{array}\right)\right)\left(\int_{0}^{1} v^{a}(1-v)^{j-a} d \zeta(v) \operatorname{Im}\left[e^{i(a+1 / 2) l}\right]\right) d l \\
& -\frac{1}{\pi} \int_{0}^{\pi}\left(\frac{\varphi_{(y)}(l)}{\sin (l / 2)} \sum_{a=0}^{j}\left(\begin{array}{c}
j \\
a
\end{array}\right)\right)\left(\int_{0}^{1} v^{a}(1-v)^{j-a} d \zeta(v) \operatorname{Re}\left[e^{i a l}\right]\right) d l
\end{aligned}
$$




$$
\begin{aligned}
= & -\frac{2 a}{\pi} \int_{0}^{\pi}\left(\frac{\rho_{(y)}(l)}{4 \sin (l / 2)}\right)\left(\int_{0}^{1} \operatorname{Im}\left[\sum_{a=0}^{j}\left(\begin{array}{c}
j \\
a
\end{array}\right) v^{a}(1-v)^{j-a} e^{i(a+1 / 2) l}\right] d \zeta(v)\right) d l \\
& -\frac{1}{\pi} \int_{0}^{\pi}\left(\frac{\rho_{(y)}(l)}{4 \sin ^{2}(l / 2)}\right)\left(\int_{0}^{1} \operatorname{Re}\left[\sum_{a=0}^{j}\left(\begin{array}{c}
j \\
a
\end{array}\right) v^{a}(1-v)^{j-a} e^{i a l}\right] d \zeta(v)\right) d l \\
= & -\frac{a}{2 \pi} \int_{0}^{\pi}\left(\frac{\rho_{(y)}(l)}{\sin (l / 2)} \int_{0}^{1} f_{1}(v, l) d \zeta(v)\right) d l-\frac{1}{4 \pi} \int_{0}^{\pi}\left(\frac{\rho_{(y)}(l)}{\sin ^{2}(l / 2)} \int_{0}^{1} f_{2}(v, l) d \zeta(v)\right) d l \\
= & \int_{0}^{\pi} \rho_{(y)}(l)\left[\tilde{Y}_{1 j}^{\prime H}(l)+\tilde{Y}_{2 j}^{\prime H}(l)\right] d l
\end{aligned}
$$

Let

$$
k_{j}^{\prime}(y):=\tilde{M}_{j}^{\prime H}(y)-\tilde{g^{\prime}}(y)=\int_{0}^{\pi} \rho_{(y)}(l)\left[\tilde{Y}_{1 j}^{\prime H}(l)+\tilde{Y}_{2 j}^{\prime H}(l)\right] d l .
$$

Then

$$
k_{j}^{\prime}(y+z)+k_{j}^{\prime}(y-z)=\int_{0}^{\pi}\left[\rho_{(y+z)}(l)+\rho_{(y-z)}(l)\right]\left[\tilde{Y}_{1 j}^{\prime H}(l)+\tilde{Y}_{2 j}^{\prime H}(l)\right] d l .
$$

Using the generalized Minkowski inequality (Chui [42]),

$$
\begin{aligned}
\left\|k_{j}^{\prime}(\cdot+z)+k_{j}^{\prime}(\cdot-z)\right\|_{r}= & \left\{\frac{1}{2 \pi} \int_{0}^{2 \pi}\left|k_{j}^{\prime}(y+z)+k_{j}^{\prime}(y-z)\right|^{r} d y\right\}^{1 / r} \\
\leq & \int_{0}^{\pi}\left\{\frac{1}{2 \pi} \int_{0}^{2 \pi}\left|\left\{\rho_{(y+z)}(l)+\rho_{(y-z)}(l)\right\}\right|^{r} d y\right\}^{1 / r}\left|\tilde{Y}_{1 j}^{\prime}(l)+\tilde{Y}_{2 j}^{\prime H}(l)\right| d l \\
= & \int_{0}^{1 /(j+1)}\left\|\rho_{(\cdot+z)}(l)+\rho_{(\cdot-z)}(l)\right\|_{r}\left|\tilde{Y}_{1 j}^{\prime}(l)+\tilde{Y}_{2 j}^{\prime H}(l)\right| d l \\
& +\int_{1 /(j+1)}^{\pi}\left\|\rho_{(\cdot+z)}(l)+\rho_{(\cdot-z)}(l)\right\|_{r}\left|\tilde{Y}_{1 j}^{\prime}{ }^{H}(l)+\tilde{Y}_{2 j}^{\prime}{ }_{j}^{H}(l)\right| d l \\
& :=I^{\prime}{ }_{1}+I^{\prime}{ }_{2} \text { (say). }
\end{aligned}
$$

Further,

$$
\left|\rho_{(y+z)}(l)+\rho_{(y-z)}(l)\right| \leq|g(y+z+l)+g(y+z-l)|+|g(y-z+l)+g(y-z-l)|
$$

Applying Minkowski's inequality (Zygmund [27]),

$$
\left\|\rho_{(\cdot+z)}(l)+\rho_{(\cdot-z)}(l)\right\|_{r} \leq\|g(\cdot+z+l)+g(\cdot+z-l)\|_{r}\|g(\cdot-z+l)+g(\cdot-z-l)\|_{r}=O(\lambda(l)) .
$$

Also

$$
\left\|\rho_{(\cdot+z)}(l)+\rho_{(\cdot-z)}(l)\right\|_{r} \leq\|g(\cdot+l+z)+g(\cdot+l-z)\|_{r}+\|g(\cdot-l+z)+g(\cdot-l-z)\|_{r}=O(\lambda(z)) .
$$

Since $v(l)$ is positive and increasing and for $l \leq z$, we have

$$
\left\|\rho_{(\cdot+z)}(l)+\rho_{(\cdot-z)}(l)\right\|_{r}=O(\lambda(l))=O\left(v(l)\left(\frac{\lambda(l)}{v(l)}\right)\right)=O\left(v(z)\left(\frac{\lambda(l)}{v(l)}\right)\right) .
$$


Since $\lambda(l) / v(l)$ is positive and increasing, and if $l \geq z$, then $\lambda(l) / v(l) \geq \lambda(z) / v(z)$ and we have

$$
\left\|\rho_{(\cdot+z)}(l)+\rho_{(\cdot-z)}(l)\right\|_{r}=O(\lambda(z))=O\left(v(z)\left(\frac{\lambda(l)}{v(l)}\right)\right) .
$$

Using (10), (31), Lemma 5, Lemma 8 and $\lambda(l) / v(l)$ being monotonic with $l$, we have

$$
\begin{aligned}
I^{\prime}{ }_{1} & =\int_{0}^{1 /(j+1)}\left\|\rho_{(\cdot+z)}(l)+\rho_{(\cdot-z)}(l)\right\|_{r}\left|\tilde{Y}_{1 j}^{\prime H}(l)+\tilde{Y}_{2 j}^{\prime H}(l)\right| d l \\
& =O\left(\int_{0}^{1 /(j+1)} v(z) \frac{\lambda(l)}{v(l)}(j+1) d l+\int_{0}^{1 /(j+1)} v(z) \frac{\lambda(l)}{v(l)} \frac{d l}{l^{2}}\right) \\
& =O\left((j+1) v(z) \frac{\lambda\left((j+1)^{-1}\right)}{v\left((j+1)^{-1}\right)} \int_{0}^{1 /(j+1)} d l\right)+O\left(v(z)(j+1) \frac{\lambda\left((j+1)^{-1}\right)}{v\left((j+1)^{-1}\right)}\right) \\
& =O\left(v(z) \frac{\lambda\left((j+1)^{-1}\right)}{v\left((j+1)^{-1}\right)}+v(z)(j+1) \frac{\lambda\left((j+1)^{-1}\right)}{v\left((j+1)^{-1}\right)}\right) .
\end{aligned}
$$

Also, using (31), Lemma 5 and Lemma 8, we have

$$
\begin{aligned}
I^{\prime}{ }_{2} & =\int_{1 /(j+1)}^{\pi}\left\|\rho_{(\cdot+z)}(l)+\rho_{(\cdot-z)}(l)\right\|_{r}\left|\tilde{Y}_{1 j}^{\prime}{ }^{H}(l)+\tilde{Y}_{2 j}^{\prime H}(l)\right| d l \\
& =O\left(\int_{1 /(j+1)}^{\pi} v(z) \frac{\lambda(l)}{v(l)} \frac{d l}{l^{2}(j+1)}\right)+O\left(\int_{1 /(j+1)}^{\pi} v(z) \frac{\lambda(l)}{v(l)} \frac{d l}{l^{3}(j+1)}\right) \\
& =O\left(\frac{v(z)}{j+1} \int_{1 /(j+1)}^{\pi} \frac{\lambda(l)}{l^{2} v(l)} d l+\frac{v(z)}{j+1} \int_{1 /(j+1)}^{\pi} \frac{\lambda(l)}{l^{3} v(l)} d l\right) .
\end{aligned}
$$

By (29), (33) and (34),

$$
\begin{aligned}
\left\|k_{j}^{\prime}(\cdot+z)+k_{j}^{\prime}(\cdot-z)\right\|_{r}= & O\left(v(z) \frac{\lambda\left((j+1)^{-1}\right)}{v\left((j+1)^{-1}\right)}+v(z)(j+1) \frac{\lambda\left((j+1)^{-1}\right)}{v\left((j+1)^{-1}\right)}\right) \\
& +O\left(\frac{v(z)}{j+1} \int_{1 /(j+1)}^{\pi} \frac{\lambda(l)}{l^{2} v(l)} d l+\frac{v(z)}{j+1} \int_{1 /(j+1)}^{\pi} \frac{\lambda(l)}{l^{3} v(l)} d l\right) .
\end{aligned}
$$

Thus,

$$
\begin{aligned}
\sup _{z \neq 0} \frac{\left\|k_{j}^{\prime}(\cdot+z)+k_{j}^{\prime}(\cdot-z)\right\|_{r}}{v(z)}= & O\left(\frac{\lambda\left((j+1)^{-1}\right)}{v\left((j+1)^{-1}\right)}+(j+1) \frac{\lambda\left((j+1)^{-1}\right)}{v\left((j+1)^{-1}\right)}\right) \\
& +O\left(\frac{1}{j+1} \int_{1 /(j+1)}^{\pi} \frac{\lambda(l)}{l^{2} v(l)} d l\right)+O\left(\frac{1}{j+1} \int_{1 /(j+1)}^{\pi} \frac{\lambda(l)}{l^{3} v(l)} d l\right) .
\end{aligned}
$$

Since $g \in Z_{r}^{(\lambda)}$, then

$$
\left\|\rho_{(\cdot)}(l)\right\|_{r}=\|g(\cdot+l)+g(\cdot-l)\|_{r}=O(\lambda(l)) .
$$

Also from (10), when $v(l)=1$, we get

$$
\int_{0}^{\eta} \frac{\lambda(l)}{l^{2}} d l=O\left(\frac{\lambda(\eta)}{\eta}\right), \quad 0<\eta<\pi .
$$


Using (35), (36), Lemma 5 and Lemma 8, we have

$$
\begin{aligned}
\left\|k_{j}^{\prime}(\cdot)\right\|_{r} & =\left(\int_{0}^{1 /(j+1)}+\int_{1 /(j+1)}^{\pi}\right)\left\|\rho_{(\cdot)}(l)\right\|_{r}\left|\tilde{Y}_{1 j}^{\prime}{ }^{H}(l)+\tilde{Y}_{2 j}^{\prime H}(l)\right| d l \\
& =\int_{0}^{1 /(j+1)}\left\|\rho_{(\cdot)}(l)\right\|_{r}\left|\tilde{Y}_{1 j}^{\prime H}(l)+\tilde{Y}_{2 j}^{\prime H}(l)\right| d l+\int_{1 /(j+1)}^{\pi}\left\|\rho_{(\cdot)}(l)\right\|_{r}\left|\tilde{Y}_{1 j}^{\prime H}(l)+\tilde{Y}_{2 j}^{\prime H}(l)\right| d l \\
& =O\left((j+1) \int_{0}^{1 /(j+1)} \lambda(l) d l+\int_{0}^{1 /(j+1)} \frac{\lambda(l)}{l^{2}} d l\right)+O\left(\frac{1}{j+1} \int_{1 /(j+1)}^{\pi} \frac{\lambda(l)}{l^{2}} d l+\frac{1}{j+1} \int_{1 /(j+1)}^{\pi} \frac{\lambda(l)}{l^{3}} d l\right) \\
& =O\left(\lambda\left((j+1)^{-1}\right)+(j+1) \lambda\left((j+1)^{-1}\right)\right)+O\left(\frac{1}{j+1} \int_{1 /(j+1)}^{\pi} \frac{\lambda(l)}{l^{2}} d l+\frac{1}{j+1} \int_{1 /(j+1)}^{\pi} \frac{\lambda(l)}{l^{3}} d l\right) .
\end{aligned}
$$

Now, we have

$$
\left\|k_{j}^{\prime}(\cdot)\right\|_{r}^{(v)}=\left\|k_{j}^{\prime}(\cdot)\right\|_{r}+\sup _{z \neq 0} \frac{\left\|k_{j}^{\prime}(\cdot+z)+k_{j}^{\prime}(\cdot-z)\right\|_{r}}{v(z)} .
$$

Using (33) and (36),

$$
\begin{aligned}
\left\|k_{j}^{\prime}(\cdot)\right\|_{r}^{(v)}= & O\left(\frac{\lambda\left((j+1)^{-1}\right)}{v\left((j+1)^{-1}\right)}\right)+O\left((j+1) \frac{\lambda\left((j+1)^{-1}\right)}{v\left((j+1)^{-1}\right)}\right)+O\left(\frac{1}{(j+1)} \int_{1 /(j+1)}^{\pi} \frac{\lambda(l)}{l^{2} v(l)} d l\right) \\
& +O\left(\frac{1}{(j+1)} \int_{1 /(j+1)}^{\pi} \frac{\lambda(l)}{l^{3} v(l)} d l\right)+O\left(\lambda\left((j+1)^{-1}\right)\right)+O\left((j+1) \lambda\left((j+1)^{-1}\right)\right) \\
& +O\left(\frac{1}{(j+1)} \int_{1 /(j+1)}^{\pi} \frac{\lambda(l)}{l^{2}} d l\right)+O\left(\frac{1}{(j+1)} \int_{1 /(j+1)}^{\pi} \frac{\lambda(l)}{l^{3}} d l\right) \\
& =\sum_{i=1}^{8} O\left(S_{i}^{\prime}{ }_{i}\right) \quad(\text { say }) .
\end{aligned}
$$

Now, we write $S^{\prime}{ }_{i}, 1 \leq i \leq 6$ in terms of $S^{\prime}{ }_{7}$ and $S^{\prime}{ }_{8}$. Since we have $\lambda(l)=\frac{\lambda(l)}{v(l)} \cdot v(l) \leq v(\pi) \frac{\lambda(l)}{v(l)}, l \in(0, \pi]$, then

$$
S_{1}^{\prime}=O\left(S_{5}^{\prime}\right) \text { and } S_{2}^{\prime}=S_{6}^{\prime}
$$

and

$$
S_{3}^{\prime}=\left(\frac{1}{(j+1)} \int_{1 /(j+1)}^{\pi} \frac{\lambda(l)}{l^{2} v(l)} v(l) d l\right) \leq\left(\frac{v(\pi)}{(j+1)} \int_{1 /(j+1)}^{\pi} \frac{\lambda(l)}{l^{2} v(l)} d l\right)=O\left(S_{7}^{\prime}\right) .
$$

Similarly,

$$
S_{4}^{\prime}=S_{8}^{\prime}
$$

Again, since $\frac{\lambda(l)}{v(l)} \leq(j+1) \frac{\lambda(l)}{v(l)}$, then

$$
S^{\prime}{ }_{5}=O\left(S_{6}^{\prime}\right)
$$


Since $\lambda(l) / \nu(l)$ is positive and increasing where $\lambda(l)$ and $v(l)$ are as in Remark 3, then

$$
\begin{aligned}
S_{8}^{\prime} & =\frac{1}{(j+1)} \int_{1 /(j+1)}^{\pi} \frac{\lambda(l)}{l^{3} v(l)} d l \geq \frac{\lambda\left((j+1)^{-1}\right)}{v\left((j+1)^{-1}\right)} \frac{1}{(j+1)} \int_{1 /(j+1)}^{\pi} \frac{d l}{l^{3}} \\
& =\frac{\lambda\left((j+1)^{-1}\right)}{v\left((j+1)^{-1}\right)} \frac{1}{(j+1)}\left[-\frac{1}{2 l^{2}}\right]_{1 /(j+1)}^{\pi}=\frac{\lambda\left((j+1)^{-1}\right)}{2 v\left((j+1)^{-1}\right)} \frac{1}{(j+1)}\left[(j+1)^{2}-\frac{1}{\pi^{2}}\right] \\
& =\frac{\lambda\left((j+1)^{-1}\right)}{2 v\left((j+1)^{-1}\right)}(j+1)\left[\frac{(j+1)^{2}-\frac{1}{\pi^{2}}}{(j+1)^{2}}\right] \geq \frac{1}{2}\left(1-\frac{1}{4 \pi^{2}}\right)(j+1) \frac{\lambda\left((j+1)^{-1}\right)}{v\left((j+1)^{-1}\right)}
\end{aligned}
$$

Then,

$$
S_{6}^{\prime}=O\left(S_{8}^{\prime}\right)
$$

From (37) to (42), we have

$$
\left\|k_{j}^{\prime}(\cdot)\right\|_{r}^{(v)}=\sum_{i=1}^{8} O\left(S_{i}^{\prime}\right)=O\left(S^{\prime}{ }_{7}\right)+O\left(S_{8}^{\prime}\right)=O\left(\frac{1}{j+1} \int_{1 /(j+1)}^{\pi} \frac{\lambda(l)}{l^{2} v(l)} d l+\frac{1}{j+1} \int_{1 /(j+1)}^{\pi} \frac{\lambda(l)}{l^{3} v(l)} d l\right) .
$$

Hence,

$$
\left\|\tilde{M}_{j}^{\prime}{ }_{j}^{H}(g, \cdot)-\tilde{g}^{\prime}(\cdot)\right\|_{r}^{v}=O\left(\frac{1}{j+1} \int_{1 /(j+1)}^{\pi} \frac{(l+1)}{l^{3}} \frac{\lambda(l)}{v(l)} d l\right)
$$

\section{Corollaries}

Corollary 1. Error approximation of a conjugate function $\tilde{g}$ of a $2 \pi$-periodic function $g \in Z_{r}^{(\lambda)}$, wherer $\geq 1$, using $H \equiv\left(\Theta_{j, a}\right)$ of CFS is given by

$$
\left\|\tilde{M}_{j}^{H}(g, \cdot)-\tilde{g}(\cdot)\right\|_{r}^{(v)}=O\left[\frac{\lambda\left(\frac{1}{j+1}\right)}{v\left(\frac{1}{j+1}\right)}(\log (j+1))\right],
$$

where $\frac{\lambda(l)}{l v(l)}$ is non-increasing.

Proof. From Theorem 1, we have

$$
\left\|\tilde{M}_{j}^{H}(g, \cdot)-\tilde{g}(\cdot)\right\|_{r}=O\left(\frac{1}{j+1} \int_{1 /(j+1)}^{\pi} \frac{\lambda(l)}{l^{2} v(l)} d l\right) .
$$

Since $\lambda(l) / l v(l)$ is non-increasing function, then

$$
\left\|\tilde{M}_{j}^{H}(g, \cdot)-\tilde{g}(\cdot)\right\|_{r}=O\left(\frac{1}{(j+1)}(j+1) \frac{\lambda(1 /(j+1))}{v(1 /(j+1))} \int_{1 /(j+1)}^{\pi} \frac{d l}{l}\right)=O\left(\frac{\lambda(1 /(j+1))}{v(1 /(j+1))}(\log (j+1))\right) .
$$

Corollary 2. Error approximation of a conjugate derived function $\tilde{g}^{\prime}$ of a $2 \pi$-periodic function $g \in Z_{r}^{(\lambda)}$, wherer $\geq$ 1 , using $H \equiv\left(\Theta_{j, a}\right)$ of CDFS is given by

$$
\left\|\tilde{M}_{j}^{\prime H}(g, \cdot)-\tilde{g}^{\prime}(\cdot)\right\|_{r}^{v}=O\left(\frac{\lambda\left((j+1)^{-1}\right)}{v\left((j+1)^{-1}\right)}((j+1)+\log (j+1))\right),
$$


where $\frac{\lambda(l)}{l v(l)}$ is non-increasing.

Proof. From Theorem 2, we have

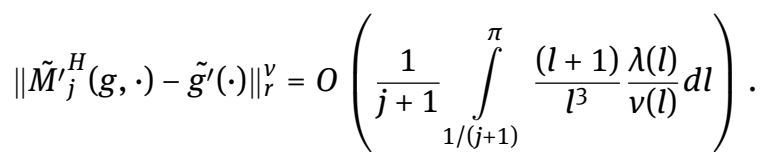

Since $\lambda(l) / l v(l)$ is non-increasing function, then

$$
\begin{aligned}
\left\|\tilde{M}_{j}^{\prime}(g, \cdot)-\tilde{g}^{\prime}(\cdot)\right\|_{r}^{v} & =O\left(\frac{1}{(j+1)}(j+1) \frac{\lambda\left((j+1)^{-1}\right)}{v\left((j+1)^{-1}\right)} \int_{1 /(j+1)}^{\pi} \frac{d l}{l}\right)+O\left(\frac{1}{(j+1)}(j+1) \frac{\lambda\left((j+1)^{-1}\right)}{v\left((j+1)^{-1}\right)} \int_{1 /(j+1)}^{\pi} \frac{d l}{l^{2}}\right) \\
& =O\left(\frac{\lambda\left((j+1)^{-1}\right)}{v\left((j+1)^{-1}\right)}((j+1)+\log (j+1))\right) .
\end{aligned}
$$

Corollary 3. Error approximation of a conjugate function $\tilde{g}$ of a $2 \pi$-periodic $g \in Z_{\alpha, r}$, where $r \geq 1$, using $H \equiv\left(\Theta_{j, a}\right)$ of CFS is given by

$$
\left\|\tilde{M}_{j}^{H}(g, \cdot)-\tilde{g}(\cdot)\right\|_{r}^{(v)}= \begin{cases}O\left((j+1)^{\alpha_{1}-\alpha_{2}}\right), & 0 \leq \alpha_{1}<\alpha_{2}<1 \\ O\left((j+1)^{-1}\{\log (j+1)\}\right), & \alpha_{1}=0, \alpha_{2}=1 .\end{cases}
$$

Proof. Putting $\lambda(l)=l^{\alpha_{2}}$ and $v(l)=l^{\alpha_{1}}$ in Theorems 1 and Corollary 1, the result follows.

\section{Particular cases}

Some particular cases of our main results are as follows:

(i) If $\lambda(l)=\xi(l)$ and $v(l)=u(l)$ in Theorem 1, then Theorem 4 of Rhoades [43] becomes a particular case of our result.

(ii) If $\lambda(l)=l^{\alpha_{2}}$ and $v(l)=l^{\alpha_{1}}$ in Theorem 1 and also if $r \rightarrow \infty$ and $\alpha_{1}=0$, then Theorem 6 of Rhoades [43] becomes a particular case of our result.

(iii) Since the Euler matrix is an $H \equiv\left(\Theta_{j, a}\right)$ matrix, and in view of remark 1, if $\lambda(l)=\xi(l)$ and $v(l)=u(l)$ in Theorem 1 and if the order of the product $(E, q)$-Hausdorff is taken as Hausdorff- $(E, q)$ (Example 1 [36, p. 68]), Theorem 3.3 of Lal and Mishra [26] becomes a particular case of our Theorem 1.

(iv) If we take $\lambda(l)=l^{\alpha_{2}}$ and $v(l)=l^{\alpha_{1}}$ in Theorem 1, where $r \rightarrow \infty$ and $\alpha_{1}=0$ and if the order of the product $(E, q)$-Hausdorff is taken as Hausdorff- $(E, q)$ (Example 1 [36, p. 68]), Theorem 3.1 of Lal and Mishra [26] becomes a particular case of our Theorem 1 .

Acknowledgments: The author expresses his gratitude towards his mother for her blessings. The author also expresses his gratitude towards his father in heaven, whose soul is always guiding and encouraging him. This research work is part of a project 25/(0225)/13/EMR-II under Council of Scientific and Industrial Research, Government of India.

The author is highly thankful to all the referees for their valuable comments and suggestions for improvement of the paper.

\section{References}

[1] Kushwaha J. K., On the approximation of generalized Lipschitz function by Euler means of conjugate series of Fourier series, Sci. World J., 2013, Article ID. 508026

[2] Nigam H. K., Sharma K., A study on degree of approximation by Karamata summability method, J. Inequal. Appl., 2011, 85(1), $1-28$ 
[3] Qureshi K., On the degree of approximation of a periodic function $f$ by almost Riesz means of its conjugate series, Indian J. Pure Appl. Math., 1982, 13(10), 1136-1139

[4] Qureshi K., On the degree of approximation of functions belonging to the Lipschitz class by means of a conjugate series, Indian J. Pure Appl. Math., 1981, 12(9), 1120-1123

[5] Qureshi K., On the degree of approximation of functions belonging to the $\operatorname{Lip}(\alpha, p)$ by means of a conjugate series, Indian J. Pure Appl. Math., 1982, 13(5), 560-563

[6] Qureshi K., On the degree of approximation to a function belonging to weighted $\left(L^{p}, \xi_{1}(t)\right)$ class, Indian J. Pure Appl. Math., 1982, 13(4), 471-475

[7] Lal S., Nigam H. K., Degree of approximation of conjugate of a function belonging to $\operatorname{Lip}(\xi(t)$, $p)$-class by matrix summability means of conjugate Fourier series, Int. J. Math. Math. Sci., 2001, 27, 555-563

[8] Lal S., On the degree of approximation of conjugate of a function belonging to weighted $W\left(L^{p}, \xi(t)\right)$ class by matrix summability means of conjugate series of a Fourier series, Tamkang J. Math., 2002, 31(4), 279-288

[9] Rhoades B. E., Hausdorff summability methods, Trans. Am. Math. Soc., 1961, 101, 396-425

[10] Mittal M. L., Singh U., Mishra V. N., Approximation of functions (signals) belonging to $W\left(L_{p}, \xi(t)\right)$-class by means of conjugate Fourier series using Nörlund operators, Varahmihir J. Math. Sci. India, 2006, 6(1), 383-392

[11] Mishra V. N., On the degree of approximation of signals (functions) belonging to the weighted $W\left(L_{p}, \xi(t)\right),(p \geq 1)$-class by almost matrix summability method of its conjugate Fourier series, Int. J. Appl. Math. Mech., 2009, 5(7), 16-27

[12] Kranz R., Lenski W., Szal B., On the degrees of approximation of functions belonging to $L^{p}(\tilde{\omega})_{\beta}$ class by matrix means of conjugate Fourier series, Math. Inequal. Appl., 2012, 15(3), 717-732

[13] Lal S., Singh H. P., The degree of approximation of conjugates of almost Lipschitz functions by $(N, p, q)(E, 1)$ means, Int. Math. Forum, 2010, 5(34), 1663-1671

[14] Lal S., Singh P. N., Degree of approximation of conjugate of $\operatorname{Lip}(\alpha, p)$ function by $(C, 1)(E, 1)$ means of conjugate series of a Fourier series, Tamkang J. Math, 2002, 33(3), 269-274

[15] Dhakal B. P., Approximation of the conjugate of a function belonging to the $W\left(L^{p}, \xi(t)\right)$ class by $\left(N, p_{n}\right)(E, 1)$ means of the conjugate series of the Fourier series, Kathmandu University Journal of Science, Engineering and Technology, 2009, 5(II), 30-36

[16] Mishra V. N., Khan H. H., Khatri K., Mishra L. N., On approximation of conjugate of signals (functions) belonging to the generalized weighted $W\left(L_{r}, \xi(t)\right),(r \geq 1)$-class by product summability means of conjugate series of Fourier series, Int. J. Math. Anal., 2012, 6(35), 1703-1715

[17] Nigam H. K., Sharma A., On approximation of conjugate of a function belonging to weighted $W\left(L_{r}, \xi(t)\right)$-class by product means, Int. J. Pure Appl. Math., 2011, 70(3), 317-328

[18] Nigam H. K., Sharma A., On approximation of conjugate of functions belonging to different classes by product means, Int. J. Pure Appl. Math., 2012, 76(2), 303-316

[19] Nigam H. K., Sharma A., On approximation of conjugate of a function belonging to $\operatorname{Lip}(\xi(t), r)$ class by product summability means of conjugate series of Fourier series, Int. J. Contemp. Math. Sci., 2010, 5(54), 2673-2683

[20] Nigam H. K., Sharma K, Approximation of conjugate of functions belonging to Lip $\alpha$ class and $W\left(L_{r}, \xi(t)\right)$-class by product means of conjugate Fourier series, Eur. J. Pure Appl. Math., 2011, 4(3), 276-286

[21] Padhy B. P., Buxi S. K., Misra M., Misra U. K., On degree of approximation by product means $(E, q)\left(\bar{N}, p_{n}\right)$ of conjugate series of Fourier series, Int. J. Math. Sci. Eng. Appl., 2002, 6(I), 363-370

[22] Rhoades B. E., A method of Hausdorff summability, Math. Z., 1963, 81, 62-75

[23] Rhoades B. E., Sharma, N. K., Spectral results for some Hausdorff matrices, Acta Sci. Math., 1982, 44, 359-364

[24] Petersen G. M., Regular Matrix Transformations, McGraw-Hill, London, 1966

[25] Singh U., Srivastava S. K., Approximation of conjugate of functions belonging to weighted Lipschitz class $W\left(L_{p}, \xi(t)\right)$ by Hausdorff means of conjugate Fourier series, J. Comput. Appl. Math. 2014, 259, 633-640

[26] Lal S., Mishra A., Euler-Hausdorff matrix summability operator and trigonometric approximation of the conjugate of a function belonging to the generalized Lipschitz class, J. Inequal. Appl., 2013, 2013:59

[27] Zygmund A., Trigonometric series, Cambridge Univ. Press, Cambridge, 3rd rev. ed., 2002

[28] Moursund A. F., On summation of derived series of the conjugate Fourier series, 1935, 36(1), 182-193

[29] Chandra P., Dikshit G. D., On the $|B|$ and $|E, q|$ summability of a Fourier series, its conjugate series and their derived series, Indian J. Pure Appl. Math., 1981, 12(11), 1350-1360

[30] Lal S., Nigam H. K., On $K^{\lambda}$-summability of derived Fourier series, Ultra Scientist of Physical Sciences, 20002, 12(3), 198-285

[31] Lal S., Yadav P., $(N, p, q)(C, 1)$ summability of derived series of a Fourier series, Bull. Col. Math. Soc., 2001, 93(2), 85-92

[32] Mursaleen M., Alotaibi A., Generalized matrix summability of a conjugate derived Fourier series, J. Inequal. Appl., 2017, 2017:273

[33] Hausdorff F., Summationsmethoden und Momentfolgen, Math. Z, 1921, 9, I:74-109, II: 280-289

[34] Boos J., Cass P., Classical and Modern Methods in Summability, Oxford University Press, New York, 2000

[35] Garabedian H. L., Hausdorff matrices, Amer. Math. Monthly, 1939, 46(7), 390-410

[36] Keska S., The degree of approximation by Hausdorff means of a conjugate Fourier series, Annales Universitatis Mariae CurieSklodowska, 2016, 70(2), 63-82 
[37] Geit V. É., On functions which are the second modulus of continuity, Izv. Vyssh. Ucheb. Zaved, Mat., 1998, 9, 38-41 [Russ. Math., 1998, 42(9), 36-38]

[38] Konyagin S. V., On the second moduli of continuity, In: Proceedings of the Stekov Institute of Mathematics, 2010, 269, 143-145

[39] Weiss M., Zygmund A., A note on smooth functions, Indag. Math., 1959, 21, 52-58

[40] Zygmund A., A remark on the integral modulus of continuity, Rev. Univ. Nac. Tucuman, 1950, 7, 259-269

[41] Singh M. V., Mittal M. L., Rhoades B. E., Approximation of functions in the generalized Zygmund class using Hausdorff means, J. Inequal. Appl., 2017, 2017:101

[42] Chui C. K., An introduction to wavelets (Wavelet analysis and its applications), Academic Press, USA, 1992, 1

[43] Rhoades B. E., The degree of approximation of functions, and their conjugates, belonging to several general Lipschitz classes by Hausdorff matrix means of the Fourier series and conjugate series of a Fourier series, Tamkang J. Math., 2014, 45(4), 389-395 